\title{
A high prevalence of abnormal personality traits in chronic users of anabolic-androgenic steroids
}

\author{
Craig J Cooper, Timothy D Noakes, Timothy Dunne, Michael I Lambert, Kevin Rochford
}

\begin{abstract}
Objective-(1) To assess the personality profiles of the anabolic androgenic steroid users (AAS) and (2) to determine whether valid premorbid personality traits could be obtained from cross sectional assessment using multisource data.

Methods-The first author became a participant-observer in a group of body builders. An experimental group of body builders who had been using AAS for no more than 18 months $(n=12)$ was identified. A group of control subjects, each of whom claimed that he did not, and never had, used AAS (n=12) was also recruited during this period. Key informants played a crucial role in recruiting subjects representative of the AAS and body building communities. An interview schedule based on the Diagnostic and statistical manual of mental disorders (DSM3-R) personality disorder criteria was conducted with each subject. Additional data were obtained from an AAS using informant and significant others including family and friends.
\end{abstract}

Results-The user group was significantly heavier than the control group and showed abnormal personality traits, in contrast to the control group. Personality traits of AAS users before the onset of not different from personality traits of control subjects. There were significant differences between the before and after personality traits in AAS user group.

Conclusions-The results suggest (1) that AAS use is associated with significant disturbances in personality profile, and (2) that these personality disturbances are possibly the direct result of AAS use. (Br f Sports Med 1996;30:246-250)

School of Education K Rochford

\section{MRC/UCT}

Bioenergetics of Exercise Research Unit, Sports Science Institute of South Africa, Newlands T D Noakes M I Lambert

Correspondence to: Professor T D Noakes, MRC/UCT Bioenergetics of Exercise Research Unit, Sports Science Institute of South Africa, Boundary Road, Newlands 7700, Republic of South Africa.

Ampetitive sport to include recreational non-competitive athletes and school children, ${ }^{23}$ who now make up the large majority of AAS users.

Although the physical and physiological effects of AAS use have been studied extensively, the real psychological and behavioural AAS use, assessed retrospectively, were

effects associated with their use are less well understood. This state of affairs may in part result from the fact that (1) most information of the psychological effects of these agents is anecdotal, and (2) most recent studies are cross sectional, so that the personality profiles of the subjects before AAS use began are not known. In addition, the effects of large non-therapeutic doses and combinations of AAS can be studied only in free living populations, as ethical constraints prevent such studies being performed under medical supervision.

Nevertheless, concurrent with the more widespread abuse of AAS has come a series of studies linking their use with marked psychiatric effects including personality disorders. ${ }^{4-6}$ Included among these disorders are the paranoid, schizotypal, antisocial, borderline, histrionic, narcissistic, and passive-aggressive personality trait disorders. These disorders are socially and personally debilitating and at times may profoundly affect the social functioning of the individual.

Accordingly, this study was designed to determine the effects of AAS use on personality traits in a group of body builders ingesting high doses of AAS in multiple combinations (stacking). Data were obtained from an interview schedule based on the Diagnostics and statistical manual of mental disorders (DSM3-R) personality disorder criteria. ${ }^{7}$ After months of observation of the subjects' behaviour, the first author and knowledgeable informants such as friends were able to validate the subjects' assessment of their own personality traits. If discrepancies occurred between the assessments of a subject, criteria were scored on the basis of consensus reached by the above persons.

The first author (CJC) became a non-AAS using member of a subgroup of bodybuilders who used AAS. This study allowed the personality traits to be observed uniquely at first hand in subjects using high doses and multiple combinations of AAS. However, a potential weakness of the study was that the premorbid personality traits of the AAS users could not be assessed directly as they had already been using AAS chronically for varying periods. Thus premorbid personality traits were assessed from historical recall of subjects and were compared to data from a group of non-using bodybuilders drawn from the same socioeconomic population and matched for age.

\section{Methods}

In order to assess the effects of anabolic steroid use on personality traits and separate underly- 
ing psychiatric symptoms from those induced by AAS exposure, it was necessary for CJC to work closely with bodybuilders from five Cape Town gymnasia. This proximity was made possible by his becoming a participant observer, by taking part in all the activities of the group, and in so doing gaining their trust. The initial infiltration was made possible by his gaining the friendship of two key individuals in the group; their friendship led to his acceptance by most of the members of the group.

As CJC's acceptance into the group increased, he was able to identify an experimental group of bodybuilders who had been using AAS for no more than 18 months $(n=12)$. Recruitment of subjects for the study occurred over a five week period. Key informants played a crucial role with respect to recruitment of subjects representative of the AAS and body building communities. A group of control subjects, each of whom claimed that he did not, and never had, used AAS $(n=12)$, was also recruited during this period.

As a result of the selection process, the 24 male subjects, aged between 17 and 26 , were recruited from an estimated population of between 400 and 600 committed body builders. Equal numbers of subjects were available in each group $(n=12)$.

Through frequent discussions during the observational period, subjects were made aware of CJC's interest in drug related research, and of his interest in their personal lives and of the possible effects of AAS on their personalities. Subjects were fully informed several weeks before the interviews of the goal of the intended interviews. In particular there was complete transparency of the intentions of the authors. Support for the proposed study was given by all the subjects for varying personal reasons including the desire to know more about themselves and the wish to participate in furthering scientific knowledge in this field.

Each subject was interviewed once for data collection. The first 20 minutes of the interviews were unstructured and were used, if necessary, to relax the subjects and to assure them of the confidential nature of the study. Interviews took place where subjects felt most relaxed, but were conducted under schedules. The interview schedules were based on the Diagnostic and statistical manual of mental disorders (DSM3-R) criteria for personality trait disorders. ${ }^{7}$ For each of the 11 personality disorders, subjects were given a list of the criteria denoting that personality trait disorder, and asked to consider carefully which if any of the criteria they satisfied. There was complete response on all the criteria by all the subjects. Moreover, subjects were consistent in nominating or denying criteria that were common to several disorders. After five months of observation of the subjects' behaviour, CJC and knowledgeable informants such as friends were able to validate the subjects' assessment of their own personality traits. If discrepancies occurred between these assessments, criteria were scored on the basis of consensus reached by the above persons.
The DSM3-R provides diagnostic criteria, which identify behaviours or traits that are characteristic of the person's recent or long term psychological functioning since early adulthood. Personality traits are enduring patterns of perceiving, relating to, and thinking about the environment and oneself, and are displayed in a wide range of important social and personal contexts. Personality disorders are diagnosed when the score for certain traits exceeds specified threshold criteria on the DSM3-R personality disorders.

Personality disorders are grouped into three clusters. Cluster A includes paranoid, schizoid, and schizotypal personality disorders and identifies persons who often appear to be odd or eccentric. Cluster B includes antisocial, borderline, histrionic, and narcissistic personality disorders. Persons with these disorders often appear dramatic, emotional, or erratic. Cluster $\mathrm{C}$ includes avoidant, dependent, obsessivecompulsive, and passive-aggressive personality disorders. Persons with these disorders often appear anxious.

Users of AAS underwent an additional second interview similar in format to the earlier interview except that subjects were asked to select, from memory, item responses for criteria within personality traits that would have applied before they began to use AAS. Special care was exercised in focusing subjects on that earlier period, such as interviewing subjects in places they frequented shortly before AAS use, as such settings facilitate memory recall. Second interviews were held within five weeks of the initial ones.

\section{STATISTICAL METHODS}

Group average scores for each personality trait were derived from the numbers of criteria for disorders satisfied by members of the control group, and of the AAS group before and after AAS use.

Paired Student's $t$ statistics were used to compare differences within the AAS user group, and the unpaired two sample Student's $t$ statistic between the control group and the AAS users. The Kruskal-Wallis test was used to evaluate differences between the control group and estimated scores of the AAS users before and after AAS use as a precaution against any unrecognised small sample violations of $t$ test assumptions. Differences were considered statistically significant for P less than 0.05 .

\section{Results}

The mean (SD) age, height, and mass of the user group was $22.3(2.5)$ years; $1.83(0.52) \mathrm{m}$ and $103(13.4) \mathrm{kg}$ respectively; and that of the controls was $20.9(2.3)$ years; $1.82(0.70) \mathrm{m}$, and $82.5(9.1) \mathrm{kg}$. Although the age and height of the two groups were similar, reported masses were greater in AAS users $(\mathbf{P}<0.05)$.

All the experimental subjects used two or more AAS concurrently on at least one occasion during the study. This activity, called stacking, was a common occurrence, the subjects often using combinations of AAS depending on what was both available and affordable. As a result combinations and 
Table 1 Average dosages and numbers of users for the different anabolic steroids used by the 12 AAS using body builders in this study

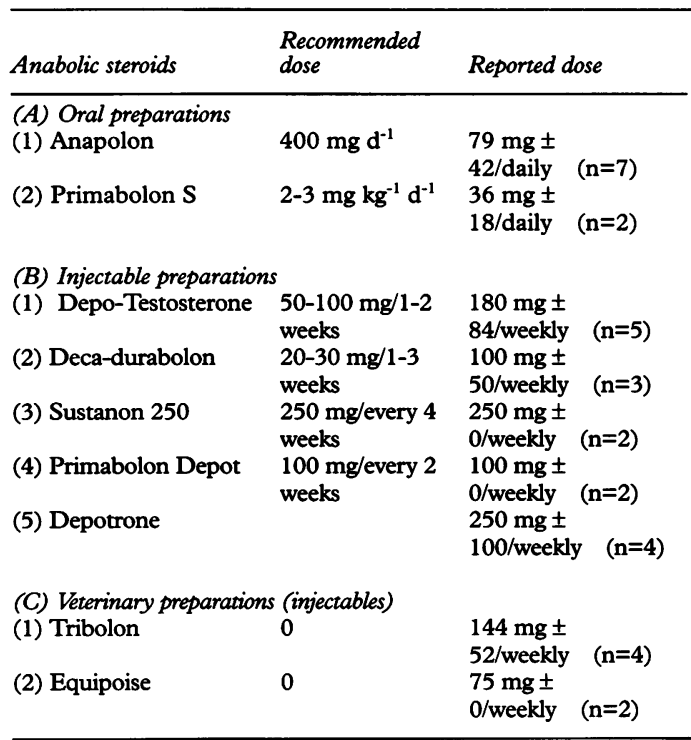

Note: Subjects used an average of 2.5 (SD 1) different drugs during any one month period. Dosages are thought to be a minimum approximation. The recommended dose for depotrone is not available as the drug has been discontinued.

dosages frequently varied each week, making it difficult to record the specified drugs and exact dosages used. All the different AAS and corresponding average dosages used over a period of one month have been listed, as accurately as possible, as the best representation of the typical pattern of AAS abuse in the user group (table 1).

Subject used an average of 2.5(1.0) AAS throughout the month. The doses used for the majority of the drugs were greatly in excess of the recommended doses used for legitimate medical reasons.

Table 2 lists the average scores and standard deviations for numbers of disorder criteria on 11 personality traits measuring eccentric, flamboyant, and anxious features of behaviour in the control and user groups. There were no statistically significant differences on these scales between the control and recall group scores (columns A and C). Four individuals (33\%) within each of these two groups showed at least one threshold criterion somewhere among these scales, though the total number of thresholds attained was higher in the AAS recall scores, ${ }^{13}$ than in the control scores. ${ }^{6}$

A twelfth variable did indicate a significant difference between control and recall scores, but was eliminated from further analysis on the basis that at least one such statistical artefact will arise by chance in a study of 12 variables with a probability of about $46 \%$. On this basis we postulate the a priori comparability of the two samples as reasonable on the remaining 11 variables.

The AAS current user scores were higher than the controls on measures of paranoid, schizoid, antisocial c, borderline, histrionic, narcissistic, and passive-aggressive personality traits (column A versus B).

On the same seven variables there was a significant difference between AAS recall and current scores. Moreover, whereas there were
Table 2 Measured traits based on counts of DSM3-R personality disorder criteria in users before and after use, and in non-users. Values are means (SD)

\begin{tabular}{|c|c|c|c|}
\hline \multirow[b]{2}{*}{$\begin{array}{l}\text { Personality } \\
\text { traits }\end{array}$} & \multirow[b]{2}{*}{$\begin{array}{l}\text { (A) Non-users } \\
\text { (control } \\
\text { group) }\end{array}$} & \multicolumn{2}{|c|}{$\begin{array}{l}\text { Users (experimental group) } \\
n=12 \text { ) }\end{array}$} \\
\hline & & $\begin{array}{l}\text { (B) Current } \\
\text { (while using } \\
A A S \text { ) }\end{array}$ & $\begin{array}{l}\text { (C) Estimated } \\
\text { (before AAS } \\
\text { use) }\end{array}$ \\
\hline Paranoid & $1.17(0.27)$ & $4.00(0.37)^{\star}$ & $1.92(0.50) \dagger$ \\
\hline Schizoid & $0.58(0.26)$ & $0.75(0.25)$ & $0.67(0.22)$ \\
\hline Schizotypal & $0.92(0.38)$ & $2.83(0.51)^{\star}$ & $1.25(0.41) \dagger$ \\
\hline Antisocial C & $0.92(0.34)$ & $3.50(0.56)^{\star}$ & $1.67(0.47) t$ \\
\hline Borderline & $0.67(0.26)$ & $3.17(0.44)^{\star}$ & $1.50(0.45) \dagger$ \\
\hline Histrionic & $1.00(0.35)$ & $2.92(0.42)^{\star}$ & $1.33(0.48) \dagger$ \\
\hline Narcissistic & $2.50(0.53)$ & $3.92(0.26)^{\star}$ & $2.08(0.40) \dagger$ \\
\hline Avoidant & $0.75(0.35)$ & $1.33(0.31)$ & $0.83(0.27)$ \\
\hline Dependent & $0.92(0.31)$ & $0.67(0.40)$ & $0.75(0.33)$ \\
\hline O. Compulsive & $1.42(0.40)$ & $2.17(0.47)$ & $1.75(0.33)$ \\
\hline P. Aggressive & $1.50(0.29)$ & $3.67(0.53)^{\star}$ & $2.17(0.55) \dagger$ \\
\hline
\end{tabular}

on recall four individuals with at least one threshold attained and two with at least two attained, there were 10 AAS users $(83 \%)$ meeting the criterion for at least one personality disorder and seven with at least two disorders on current scores.

\section{Discussion}

Research into personality assessment is fraught with difficulty. ${ }^{89}$ Serious issues to the validity of data were (1) the unknown validity of standardised self report questionnaires such as the PDQ-R and SCID 2, and (2) intentional or unintentional underreporting or over reporting by subjects on self report questionnaires.

Despite the fact that instruments are essentially a direct translation of DSM3-R personality disorder diagnostic criteria into a self report format, it is unclear how representative each question is of its respective criterion. The resulting problem of data reliability may be illustrated by comparison of any two instruments used in the same subject, which reveals on average more diagnostic disagreement than agreement. $^{8-10}$ The problem is further amplified because each subjects' interpretation of a question may be somewhat idiosyncratic. ${ }^{8}$

Intentional misreporting includes lying and efforts to meet sociably acceptable standards. ${ }^{112}$ Unintentional misreporting includes state effects and personality traits. Misreporting may be a problem for data validity' as AAS users satisfying the diagnostic number of criteria for the paranoid disorder were reluctant to fill in self report measures in this study. Even when interviewed they were likely to deny or minimise their disorder. On the other hand AAS users with the antisocial or borderline disorder were inclined to overreport or embellish their disorder.

It is increasingly realised, in the light of the above, that assessment using multisource data, particularly those from observation and significant others, is important if valid data are to be obtained. ${ }^{8}$ Hence the methodological design of this study.

AAS are psychoactive drugs which alter mood and awareness of the external (surroundings) and internal (cognitive) environ- 
ments of an individual. ${ }^{17}$ It might therefore be expected that large doses of these agents could alter personality traits in which individuals respond to other individuals and situations. These changes may then lead to abnormal personality traits or personality disorders, which then interfere with personal functioning or cause the individual to become distressed.?

Our study supports this thesis by showing increased scores in AAS users in seven personality traits (table 2). These scores are in general higher than those for controls and for historically reported values for the same AAS users before the start of AAS use.

Although some studies have reported relatively minor psychological effects of AAS use, ${ }^{12}$ other more recent studies have identified more psychological and behavioural changes associated with their use. ${ }^{4-61314}$. Thus Yates et $a l^{6}$ found that $85 \%$ of 20 AAS users showed personality disorders or abnormal personality traits on the personality disorder questionnaire (PDQ). Our results are similar (83\%). Similarly, although the findings of Perry and Hughes ${ }^{14}$ were not as clear and marked as in the aforementioned studies, they found that AAS users showed more personality disturbances overall than a control group of nonAAS users. Su et alt also found a significant impact on personality even during short term, relatively low dose AAS use without stacking.

The issue of publication bias arises in such cases. Journal articles are generally required to show statistically significant results before being accepted for publication. Thus studies which might give contrasting evidence of an absence of effects never reach a wider readership. In this instance we have not been able to trace any such studies. Furthermore, we believe that the interpretation of AAS as a cause of behavioural change is reasonable and not an artefact of publication bias.

In general the interpretation of most studies of the purported effects of AAS use on personality has been hampered by the absence of information about premorbid personality traits in current AAS users. For example, an individual may satisfy the diagnostic number of criteria for a personality disorder before AAS exposure. AAS use may exacerbate the disorder. If no premorbid data are available, the disorder may be attributed to the effects of AAS abuse. This notion is corroborated by the finding that one in three users (33\%) in this study satisfied the diagnostic number of criteria for at least one disorder before AAS exposure. In contrast, no such abnormalities were present in the control group. The possibility that personality disorders may predispose to AAS use should be considered.

Furthermore retrospective assessment of personality traits is problematic. The few studies which have attempted to control for premorbid personality traits had non-AASusing controls to determine an average premorbid profile. ${ }^{413}$ However, because a drug effect is dependent on the interaction of various influences in a particular context, there can be a wide variation in the manner in which different individuals respond to the same drugs. In addition, because of varying influences affecting drug responses, psychological effects may vary between different socioeconomic settings, different AAS and dosages used, and between different AAS using communities. Hence, if these factors are not controlled, choosing of non-AAS-users as subjects to control for premorbid traits in the AAS using community may not be valid.

The controls in this study were carefully matched for socioeconomic background and age. There were no significant differences in personality disorders between the control and user group before AAS exposure. We suggest that cross sectional assessment using multisource data was successful in assessing the premorbid personalities of users, despite major methodological problems.

The importance of controlling for premorbid personality traits cannot be overemphasised because effective diagnosis, intervention, and treatment in a clinical setting may depend on knowing which disorders or symptoms are a natural part of the patient's personality and which are induced by substance abuse.

In conclusion, this study suggests (1) that AAS use significantly increases the frequency and severity of symptoms of personality disorders; and (2) that cross sectional assessment using multisource data may allow valid retrospective assessment of premorbid personality profiles. This study adds to the growing evidence of the dangers of AAS use and suggests that observation, and information obtained from significant others, can be important in the retrospective assessment of personality. $8-1015$ The authors with to thank Drs Bryan Dirks and Elanor Nash of the Department of Psychiatry, University of Cape Town and

This study was supported by the Medical Research Council and the Nellie Atkinson and Harry Crossley Research Funds of the University of Cape Town.

1 Kaskin KB, Klebber HD. Hooked on hormones? An anabolic steroid addiction hypothesis. $\mathcal{F} A M A$ 1989;262:3166-70.

2 Buckley WE, Yesalis CE, Anderson W, Streit AL, Wright J. Estimated prevalence of anabolic steroid use among male high school seniours. $¥ A M A 1988 ; 260: 3441-5$.

3 Schwellnus MP, Lambert MI, Todd MP, Juritz JM. Androgenic anabolic steroid use in matric pupils. A survey of genic anabolic steroid use in matric pupils. A survey of
prevalence of use in the Western Cape. S Afr Med $\mathcal{1} 1992$;

82:154-8.
Pope H, Katz DL. Affective and psychotic symptoms associated with anabolic steroid use. Am F Psychiatry 1988, 145:487-90

5 Su TP, Pagliaro M, Schmidt PJ, Pickar D, Wolkowitz O Rubinow DR. Neuropsychiatric effects of anabolic steroids in male normal volunteers. $\mathscr{f} A M A$ 1993;296:2760-4.

6 Yates WR, Perry PJ, Anderson KH. Illicit anabolic steroid use: a controlled personality study. Acta Psychiatr Scand 1990;81:548-50.

7 American Psychiatric Association. Diagnostic and statistical manual of mental misorders. Washington DC:American Psychiatric Association, 1987.

8 Perry JC. Problems and considerations in the valid assessment of personality disorders. Am $\mathcal{F}$ Psychiatry 1992; 149:1645-53.

9 Hyler SE, Rieder RO, Williams JBW, Spitzer RL, Lyons M, Hendler JA. Comparison of clinical and self-report diagnosis of DSM-3 personality disorders in 555 patients. Compr

0 Hyler SE, Skodal AE, Kellman HD, Oldham JM, Rosnick L. Validity of the personality questionnaire - revised: Comparison with two structured interviews. Am $\mathcal{F}$ Psychiatry 1990;147:1043-8.

11 Yesalis CE. Incidence of anabolic steroid use: a discussion of methodological issues. In Yesalis CE, ed. Anabolic steroids in sport and exercise. Champaign, Illinois: Human Kinetics Publishers, 1993:49-70. 
12 Brower K, Eliopulos GA, Blow FC, Catlin DH, Beresford TP. Evidence for physical and psychological dependence on anabolic androgenic steroids in eight weight lifter. $A m \mathcal{F}$ Psychiatry 1990;147:510-2.

13 Bahrke MS, Wright JE, Strauss RH, Catlin DH. Psychological moods and subjectivity perceived behavioural and somatic changes accompanying anabolic-androgenic steroid use. Am F Sports Med 1993:20:717-22.
14 Perry H, Hughes G. A case of affective disorder associated with the misuse of "anabolic steroids". Br $\mathcal{F}$ Sports Med 1992;26:219-20.

15 Reis R. Co-occurance of substance abuse disorders, psychiatric disorders-separation "an art". Psycholink Aug 8 1993.

\section{Clinical tests in sports medicine: more on Achilles tendon}

The diagnosis of Achilles tendon rupture can be difficult even for experienced practitioners, and a significant number of patients can be initially misdiagnosed.

When examined soon after the injury, a tear in the substance of the tendon can be seen and palpated. With increased time after the tear, the gap can be obliterated by oedema, and palpation becomes unreliable.

In addition to the tests described in this journal in the June issue (volume 30 , page 124), two other clinical tests should be mentioned.

\section{Matles test}

The patient, lying prone and with feet over the end of the examination couch, is asked to flex the knee actively to $90^{\circ}$. The position of the ankle and feet is observed during this movement. If the foot on the affected side falls into neutral or into dorsiflexion, an Achilles tendon rupture is diagnosed. In normal patients, the foot remains in slight planter flexion when the knee is flexed to $90^{\circ} .{ }^{1}$

\section{Needle test}

With the patient prone, a small gauge needle is inserted at a right angle through the skin of the calf, just medial to the midlline, $10 \mathrm{~cm}$ proximal to the superior border of the calcaneum. The needle should be inserted only until the tip is just within the substance of the tendon. The foot is then passively and alternately dorsiflexed and plantarflexed. Two responses may occur. The needle may swivel so that it points in the direction opposite to the motion of the ankle (that is, when the ankle is dorsiflexed, the needle points distally). This indicates that the tendon is intact in the portion distal to the needle insertion point. The other possible response is that the needle does not move, or moves slightly in the same direction of the motion of the ankle (that is, when the ankle is dorsiflexed, the needle points proximally). In this second instance, the Achilles tendon has lost its continuity between the needle and its insertion. ${ }^{2}$

\section{More on the calf squeeze test}

Given the history of the test, the correct denomination should be Simmond's test. Simmonds described his test in a paper on the diagnosis of Achilles tendon rupture, published five years before Thompson's papers. ${ }^{3}$ Thompson and Doherty published jointly an article in an American journal solely on this test in $1962 .{ }^{4}$ Practically the same paper was published by Thompson, without his colleague, some months later in a European journal. ${ }^{5}$

In the British Isles, the calf squeeze test is called "Simmond's test", and in most of the rest of the world "Thompson's test", not acknowledging that Doherty was one of the original authors. To avoid offending the sensitivities of British or American influenced surgeons, it is better to describe the test as the "calf squeeze test" and cite the fact that Simmonds described it well before Thompson and Doherty. To avoid confusion on what constitutes a positive test, one should probably just mention whether the ankle plantar flexes on squeezing the calf muscles. If it does, the tendon is most likely intact (but beware of the plantaris!).

NICOLA MAFFULLI

1 Matles AL Rupture of the tendo Achilles. Another diagnostic test. Bull Hosp foint Dis 1975;36:48-51.

O'Brien T The needle test for complete rupture of the Achilles tendon. 7 Bone foint Surg Am 1984;66:1099-101 Achilles tend Simmonds FA The diagnosis of

tendon. Practitioner 1957;179;56-8 Thompson TC, Doherty JC. Spontaneous rupture of the Achilles tendo

1962;2:126-9. A test for rupture of the tendo Achillis. Acta Orthop Scand 1962;32:4615. 